Draft Version OCTOBER 28, 2018

Preprint typeset using $\mathrm{LAT}_{\mathrm{E}} \mathrm{X}$ style emulateapj v. 11/12/01

\title{
CONSTRAINING THE STAR FORMATION HISTORIES OF GRB HOST GALAXIES FROM THEIR OBSERVED ABUNDANCE PATTERNS
}

\author{
Francesco Calura ${ }^{1}$, Miroslava Dessauges-Zavadsky ${ }^{2}$, Jason X. Prochaska ${ }^{3}$ and \\ FRANCESCA MATTEUCCi ${ }^{1}$ \\ 1 Dipartimento di Astronomia-Universitá di Trieste, Via G. B. Tiepolo w 11, 34131 Trieste, Italy \\ 2 Observatoire de Geneve, Universite de Geneve, 51 Ch. des Maillettes, 1290 Sauverny, Switzerland \\ 3 UCO/Lick Observatory, University of California at Santa Cruz, Santa Cruz, CA 95064, USA \\ fcalura@oats.inaf.it \\ Draft version October 28, 2018
}

\begin{abstract}
Long-duration Gamma Ray Bursts (GRBs) are linked to the collapse of massive stars and their hosts are exclusively identified as active, star forming galaxies. Four long GRBs observed at high spectral resolution at redshift $1.5 \leq z \leq 4$ allowed the determination of the elemental abundances for a set of different chemical elements. In this paper, for the first time, by means of detailed chemical evolution models taking into account also dust production, we attempt to constrain the star formation history of the host galaxies of these GRBs from the study of the measured chemical abundances measured in their ISM. We are also able to provide constraints on the age and on the dust content of GRB hosts. Our results support the hypothesis that long duration GRBs occur preferentially in low metallicity, star forming galaxies. We compare the specific star formation rate, namely the star formation rate per unit stellar mass, predicted for the hosts of these GRBs with observational values for GRB hosts distributed across a large redshift range. Our models predict a decrease of the specific star formation rate (SSFR) with redshift, consistent with the observed decrease of the comoving cosmic SFR density between $z \sim 2$ and $z=0$. On the other hand, observed GRB hosts seems to follow an opposite trend in the SSFR vs redshift plot, with an increase of the SSFR with decreasing redshift. Future SSFR determination in larger samples of GRB hosts will be important to understand whether this trend is real or due to some selection effect. Finally, we compare the SSFR of GRB050730 host with values derived with a sample of Quasar damped Lyman alpha systems. Our results indicate that the abundance pattern and the specific star formation rates of the host galaxies of these GRBs are basically compatible with the ones determined for a sample of Quasar damped Lyman alpha systems, suggesting similar chemical evolution paths.
\end{abstract}

Subject headings: Gamma rays: burst. Galaxies: high-redshift. Galaxies: abundances; interstellar medium.

\section{INTRODUCTION}

Gamma-ray bursts (GRBs) are valuable tools to investigate the high-redshift Universe. The extreme luminosities of their afterglows have allowed detailed studies of the physical properties of the interstellar medium (ISM) of their host galaxies. In particular, in a few cases, the determination of their chemical abundance pattern has been possible (Savaglio et al. 2003; Vreeswijk et al. 2004; Prochaska et al. 2004; Penprase et al. 2006; Levesque \& Kewlev 2007; Wiersema et al. 2007; Prochaska et al. 2007a), as well as the determination of a few quantities related to their host galaxies, such as their dust content, their stellar mass and their star formation rate (Bloom et al. 1998; Le Floc'h et al. 2002; Diorgovski et al. 2003; Christensen et al. 2004; Le Floc'h et al. 2006; Castro Cerón et al. 2006; Savaglio et al. 2008).

Chen et al. (2005) first reported on the chemical abundances for the damped Lyman Alpha system (DLA) associated with the host galaxy of GRB 050730 . Their analysis showed this gas was metal poor with a modest depletion level (see also Starling et al. 2005). These results were subsequently expanded and tabulated by Prochaska et al. (2007a) (hereafter P07, see also D'Elia et al 2007) and we adopt their values adjusted to the solar relative abundances of Grevesse et al. (2007).
In this paper, for the first time we aim at determining the star formation history of some GRB DLA systems by analysing the chemical abundances measured in their afterglow spectra. For this purpose, we use a detailed chemical evolution model, which allows us to predict the evolution of the abundances of the chemical elements studied in the GRB DLAs of P07. Our aim is to constrain several relevant astrophysical quantities, such as the star formation rates, the dust content and the ages of the host galaxies of these GRBs. Our approach has already turned out to be fruitful to study Quasar DLAs on the basis of their chemical abundance pattern, allowing us to derive crucial information on their nature and on their physical properties (Dessauges-Zavadsky et al. 2004, 2007). The plan of this paper is as follows. In Section 2, we briefly introduce the chemical evolution model. In Section 3, we present our results and in Section 4 we draw some conclusions.

\section{THE CHEMICAL EVOLUTION MODEL}

\subsection{The dwarf irregular model}

The model used to derive the star formation histories of these GRB DLAs host galaxies is similar to that developed by Bradamante et al. (1998) to study dwarf irregular galaxies. Since some chemical species studied in this paper are refractory $(\mathrm{C}, \mathrm{O}, \mathrm{Si}, \mathrm{Mg}, \mathrm{Fe}, \mathrm{Ni})$, in the chemical evolution model used here we include also a detailed 
Calura et al.

treatment of dust production and destruction following the work of Calura et al. (2008) (CPM08, see section 2.2). We choose to use the model for dwarf irregular galaxies since several observational investigations provided strong evidence that most of the GRBs originate in gas rich, star-forming sub-luminous $\left(L<L_{*}\right)$ galaxies with relatively low metallicities $\left(Z<Z_{\odot}\right)$ (Bloom et al. 1998; Fruchter et al. 1999; Prochaska et al. 2004; Vreeswijk et al. 2004).

The dwarf galaxy is assumed to form by means of a continuous infall of pristine gas until a mass $M_{t o t}$ is accumulated. The evolution of dwarf irregular galaxies is characterized by a continuous star formation history. The star formation rate $\psi(t)$ is directly proportional the gas fraction $G(t)$ at the time $t$, according to the Schmidt law (Schmidt 1959)

$$
\psi(t)=\nu G(t) \text {. }
$$

where $\nu$ is the efficiency of star formation. We take into account the energy feedback from both Type Ia and Type II supernovae (SNe). They are responsible for the onset of a galactic wind, when the thermal energy of the ISM exceeds its binding energy, which is related to the presence of a dark matter halo in which the galaxy is embedded (for more details, see Bradamante et al. 1998, Lanfranchi \& Matteucci 2003). The thermal energy of the gas is controlled mainly by the thermalization efficiencies of the supernovae explosions $\left(\eta_{S N I I}\right.$ for SNe II and $\eta_{S N I a}$ for SNeIa) and stellar winds $\left(\eta_{S W}\right)$, which control the fraction of the energy restored to the ISM which is converted to thermal energy of the gas (see Bradamante et al. 1998). The binding energy of the gas, on the other hand, is strongly influenced by assumptions concerning the presence and distribution of dark matter (Matteucci 1992). A diffuse $\left(R_{e} / R_{d}=0.1\right.$, where $R_{e}$ is the effective radius of the galaxy and $R_{d}$ is the radius of the dark matter core) but relatively massive $\left(M_{\text {dark }} / M_{\text {Lum }}=10\right)$ dark halo has been assumed for each galaxy. The time at which the wind develops depends on the assumed star formation efficiency. In general, the higher the SF efficiency, the earlier the wind develops.

No instantaneous recycling approximation is adopted, i.e. the stellar lifetimes are properly taken into account. This allows us to compute in detail the contributions by low mass stars, Type Ia, Type II SNe to the chemical enrichment of the ISM. The main physical quantities related to these processes are the stellar yields, representing the fractions of processed matter restored by the stars into the ISM. The stellar yields used in this work are from Meynet \& Maeder (2002) for low and intermediate mass stars (LIMS), from Woosley \& Weaver (1995) for massive stars and from Iwamoto et al. (1999) for Type Ia SNe. We assume a Salpeter initial mass function (IMF). With such an IMF, it is possible to reproduce the chemical abundances and the observational features of dwarf irregulars (Calura \& Matteucci 2006) and to account for the local metal budget (Calura \& Matteucci 2004). We assume a cosmological model characterized by $\Omega_{m}=0.3, \Omega_{\Lambda}=0.7$ and a Hubble constant $H_{0}=70 \mathrm{kms}^{-1} M p c^{-1}$.

\subsection{N nucleosynthesis: indications from QSO DLAs}

Although N nucleosynthesis is still a matter of debate, most of $\mathrm{N}$ production arise from low and intermediate mass stars since massive stars produce only a small fraction of it (e.g. Chiappini, Matteucci \& Ballero 2005). In particular, in the solar neighborhood the typical timescale for $\mathrm{N}$ enrichment should be 0.25-0.30 Gyr. However, the N produced in massive stars is important for understanding $\mathrm{N}$ abundances at very low metallicities. In principle, $\mathrm{N}$ produced in massive stars should be secondary, namely produced by means of the $\mathrm{CNO}$ cycle starting from the $\mathrm{C}$ and $\mathrm{O}$ abundances originally present in the star. In the last years Meynet \& Maeder (2000, 2002) and more recently Chiappini et al (2006) have shown that very metal poor stars can be fast rotators and that rotation can produce primary $\mathrm{N}$, namely $\mathrm{N}$ originating from the $\mathrm{C}$ and $\mathrm{O}$ produced by the star, not those originally present in the star at birth. In this case, a high $\mathrm{N}$ abundance is expected at very low metallicities. It is not clear whether this high production of primary $\mathrm{N}$ in massive stars continue at intermediate metallicities. In our case we adopt the standard prescriptions for secondary $\mathrm{N}$ in massive stars and secondary plus primary $\mathrm{N}$ in low and intermediate mass stars.

In Fig. 1, we show the predicted evolution of $\log (\mathrm{N} / \mathrm{O})$ vs time (upper panel) and vs metallicity (lower panel) assuming various star formation efficiencies. The models are computed with the nucleosynthesis prescriptions used here (thick lines) and assuming primary $\mathrm{N}$ in massive stars (thin lines), following Matteucci (1986). The predictions are compared with the observed $\mathrm{N} / \mathrm{O}$ values measured in high redshift QSO DLAs by various authors (Pettini et al. 2008 and references therein). The prescriptions adopted here are in good agreement with the most recent data published by Pettini et al. (2008) for DLAs , whose metallicities are not as low as metal poor halo stars where a high $\mathrm{N}$ abundance seems to be found (Spite et al. 2005). The assumption of primary $\mathrm{N}$ in massive stars predicts high $\mathrm{N} / \mathrm{O}$ values at low metallicity, i.e. immediately after the beginning of star formation. However, as can be seen from Fig. 1, the highest $\mathrm{N} / \mathrm{O}$ values in DLAs can be explained also without assuming primary $\mathrm{N}$ production in massive stars, but by assuming a low star formation efficiency.

Without primary $\mathrm{N}$ production in massive stars, the value $\log (\mathrm{N} / \mathrm{O})=-2.6$, corresponding to the lowest value observed in DLAs, is reached by our models after $0.05 \mathrm{Gyr}$ with $\nu=0.01 \mathrm{Gyr}^{-1}$ and after $0.1 \mathrm{Gyr}$ with $\nu=1 \mathrm{Gyr}^{-1}$. Finally, it is worth stressing that, as remarked also by Chiappini et al. (2003), the stellar yields of Meynet \& Maeder (2002) do not include the third dredge-up and hot-bottom burning, thus their $\mathrm{N}$ yields for intermediate mass stars should be regarded as lower limits.

\subsection{Dust production and destruction}

The model for dust evolution used in this paper adopts the same prescriptions as CPM08. For a detailed description of the dust evolution model, we address the reader to that paper. Here, we briefly summarize our assumptions. For the refractory chemical element labeled $i$, a fraction $\delta_{i}^{S W}, \delta_{i}^{I a}$, and $\delta_{i}^{I I}$ is incorporated in dust grains by low and intermediate mass stars, type Ia SNe, and type II SNe, respectively. These quantities are the dust condensation efficiencies of the element $i$ in various stellar objects. Here we assume $\delta_{i}^{S W}=\delta_{i}^{I a}=\delta_{i}^{I I} \equiv \delta_{i}=0.1$. This choice is supported by recent mid-infrared observations of one 
Supernova (Sugerman et al. 2006), which provided an upper limit of $\delta_{i}^{I I} \leq 0.12$. The value assumed here is also supported by theoretical studies of the local dust cycle (Edmunds 2001). Our assumption is compatible with values provided by other theoretical and observational studies (see Morgan \& Edmunds 2003). However, other studies suggest that the dust condensation efficiencies of SNe may be even lower, with values ranging from $\sim 2 \times 10^{-4}$ to 0.15 (Zhukovska et al. 2008).

CPM08 showed that the dust depletion pattern is strongly dependent on the star formation history. The depletion pattern observed in galaxies of different morphological types provides us with the most complete set of dust depletion data for a range of different star formation histories. The refractory elements involved in this study are C and $\mathrm{O}$ which in the local ISM show mild depletion levels, and $\mathrm{Mg}, \mathrm{Si}, \mathrm{Ni}$, and $\mathrm{Fe}$, which locally show higher depletion levels (Savage \& Sembach 1996). A debate is currently open on the nature of S and on whether it may be incorporated into dust grains or not. Observational studies of the depletion patterns of the cold ISM of the Milky Way report for $\mathrm{S}$ depletion values between 0 and -0.2 dex Welty et al. 1999a). More recent data, based on observational determinations of the gas-phase abundances of some chemical elements in the Local Interstellar Cloud, support a considerable depletion for S of -0.3 dex (Kimura et al. 2003). However, these high depletion values have been derived by assuming as cosmic $\mathrm{S}$ abundance the solar value observed by Grevesse \& Sauval (1998), i.e. $A_{S}=21.4$ per $10^{6}$ hydrogen atoms. The recent update by Grevesse et al. (2007) suggests a solar $\mathrm{S}$ abundance $A_{S}=14$ per $10^{6}$ Hydrogen Atoms, which implies a S dust fraction in the Local Interstellar Cloud lower than 0.4, i.e. a logarithmic depletion higher than -0.2 dex. However, other indirect arguments indicate that S may have a refractory nature. Detailed models of dark insterstellar clouds chemistry suggest that sulphur must be depleted by two to three orders of magnitude in dark regions (Ruffle et al. 1999; Scappini et al. 2003). A recent investigation of dust and element abundances in planetary nebulae has shown that in these systems, depletion is likely to be severe for various species, including S (Phillips 2007). In cometary dust particles, $\mathrm{S}$ is present in iron sulphide $(\mathrm{FeS})$ grains (Lodders 2003). A broad feature ascribed to FeS emission has been recently seen in the infrared spectra of young stellar objects (Keller et al. 2002). Furthermore, an indirect evidence of the fact that S may suffer depletion comes from the fact that its condensation temperature is considerably higher than the ones of $\mathrm{C}$ and $\mathrm{O}$, which are known to be depleted in the gas phase (see Welty et al. 1999). From a photospheric abundance study of a RV Tauri star in the Large Magellanic Cloud, Reyniers \& Van Winckel (2007) showed how the element abundance anti-correlates with the condensation temperature, possibly implying a correlation between the depletion and the condensation temperature. In the light of these facts, in our study we consider two different scenarios for dust grain composition. In the "expected dust" scenario, we assume that the only elements going into dust grains are $\mathrm{Ni}$ and $\mathrm{Fe}$. The reason of the name chosen for this scenario lies in the fact that mainly indirect evidences indicate that $\mathrm{S}$ could be a refractory element. We consider also an "alternative dust" scenario, where also $\mathrm{S}$ is refractory.

Dust grains can be destroyed by the propagation of supernova shock waves in the warm/ionised interstellar medium (McKee 1989; Jones et al. 1994). If $G_{d u s t, i}$ is the fraction of the element $i$ locked into dust and $G$ is the gas fraction, the destruction rate is calculated as $G_{d u s t, i} / \tau_{\text {destr }}$, where $\tau_{\text {destr }}$ is the dust destruction timescale, calculated as:

$$
\tau_{\text {destr }}=\left(\epsilon M_{S N R}\right)^{-1} \frac{G}{R_{S N}} .
$$

(McKee 1989; Dwek 1998). $\quad M_{S N R}=1300 M_{\odot}$ is the mass of the interstellar gas swept up by the SN remnant (McKee 1989; Dwek et al. 2007). $R_{S N}$ is the total SNe rate, i.e. the sum of the rates of Type Ia and Type II SNe. Unless otherwise specified, we assume that no dust accretion is taking place in the GRB host galaxies. This phenomenon occurs in $\mathrm{H}_{2}$-rich molecular clouds (Dwek 1998; Calura et al. 2008). Our choice is motivated by the fact that very little molecular $\mathrm{H}$ is observed in local dwarfs, with molecular-to-atomic gas fractions of $\sim 10 \%$ or lower (Lisenfeld \& Ferrara 1998; Clavton et al. 1996). Our assumption is further supported by the fact that a very little amount of $\mathrm{H}_{2}$ observed in the spectra of GRB afterglows (Vreeswijk et al. 2004; Fvnbo et al. 2006; Tumlinson et al. 2007; Whalen et al. 2008). This might indicate that in the presence of intense SF, molecular clouds could rapidly dissolve, likely allowing very little dust accretion to occur. We will test the reliability of this assumption while discussing our results.

\section{RESULTS}

In this section, we attempt to constrain the star formation histories for the set of 4 GRB host galaxies. The abundance ratios measured by P07 for the 4 GRB hosts are presented in Table 1, as well as the predicted abundances computed by means of the best model, when possible.

We address the reader to the paper by Prochaska et al. (2007a) for further details on the GRB observations and on the techniques used to derive the ISM abundances of the GRB host galaxies.

\subsection{Abundance ratios as a diagnostic to infer the star formation history}

In chemical evolution models, the abundance ratios between two elements formed on different timescales can be used as "cosmic clocks" and provide us with information on the roles of LIMS and SNe in the enrichment process (Matteucci 2001). In particular, the study of abundance ratios such as $[\alpha / \mathrm{Fe}]$ and $[\mathrm{N} / \alpha] \mathrm{1}$ is quite useful, since the $\alpha$-elements $(\mathrm{O}, \mathrm{S}, \mathrm{Mg})$ are produced on short timescales by Type II SNe, whereas the Fe-peak elements and nitrogen are produced on long timescales by Type Ia SNe and low and intermediate-mass stars, respectively. Calura et al. (2003); Dessauges-Zavadsky et al. (2004, 2007) have shown that the simultaneous study of the abundance ratios between different elements as functions of a

${ }^{1}$ All the abundances between two different elements $\mathrm{X}$ and $\mathrm{Y}$ are expressed as $[X / Y]=\log (X / Y)-\log (X / Y) \odot$, where $(X / Y)$ and $(X / Y) \odot$ are the ratios between the mass fractions of $\mathrm{X}$ and $\mathrm{Y}$ in the ISM and in the sun, respectively. We use the set of solar abundances as determined by Grevesse et al. (2007). 

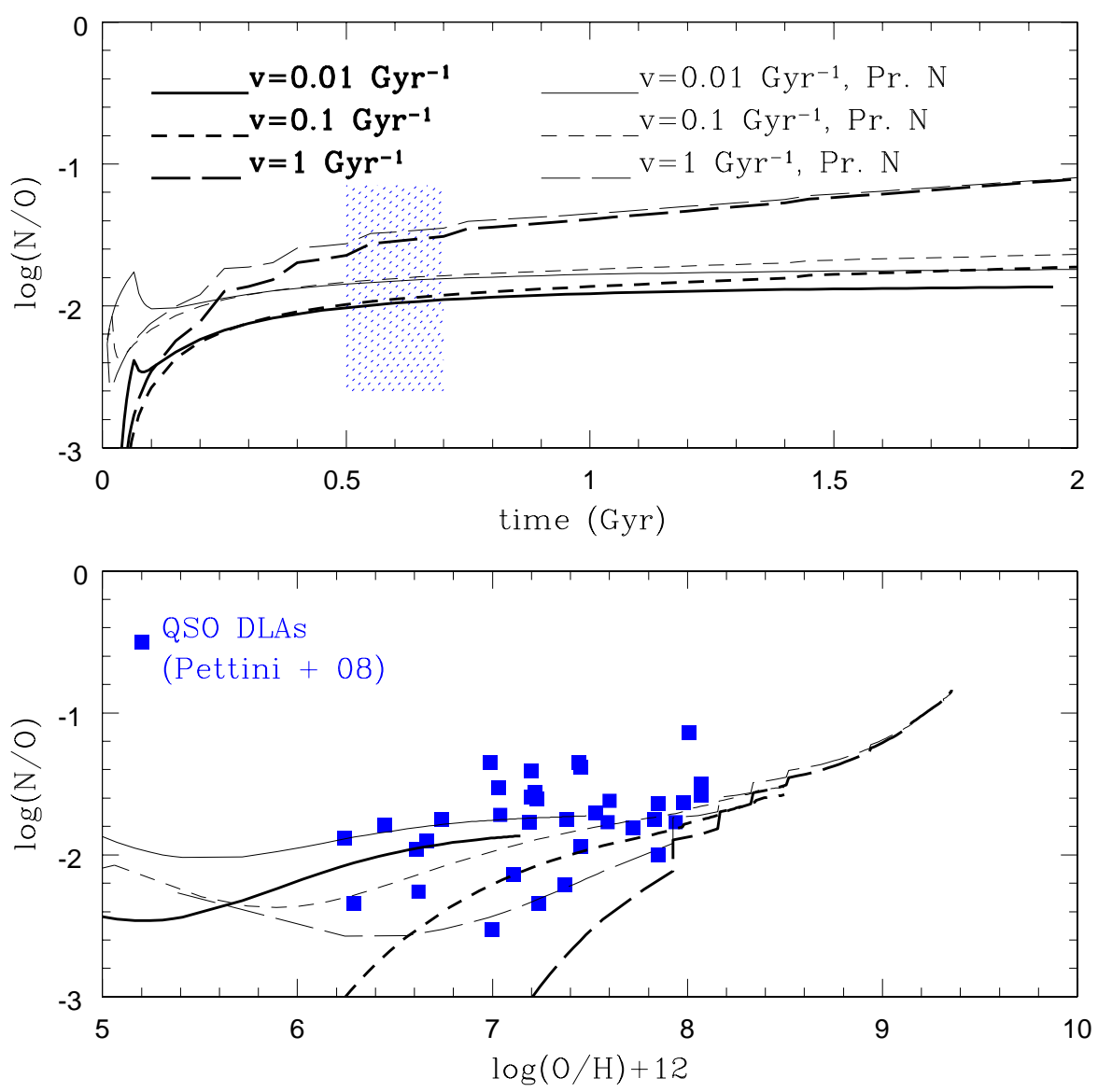

FIG. 1.- Upper panel : Predicted $\log (\mathrm{O} / \mathrm{N})$ vs time for models adopting various star formation efficiencies. The thin lines include primary $\mathrm{N}$ in massive stars following Matteucci (1986). The thick lines do not include primary $\mathrm{N}$ production in massive stars. The shaded area represents the range of $\log (\mathrm{N} / \mathrm{O})$ values observed by various authors (Pettini et al. 2008 and references therein) in a sample of QSO Damped Lyman Alpha systems. Lower panel: Predicted $\log (\mathrm{O} / \mathrm{N})$ vs $\log (\mathrm{O} / \mathrm{H})+12$. Thick and thin lines as in the upper panel. The solid squares are observational values in high-redshift QSO DLAs (Pettini et al. 2008 and references therein).

TABLE 1

\begin{tabular}{lcccccccc}
\hline Ab. ratio & \multicolumn{2}{c}{ GRB 050730} & \multicolumn{2}{c}{ GRB 050820} & \multicolumn{2}{c}{ GRB 051111 } & \multicolumn{2}{c}{ GRB 060418 } \\
\hline \hline & Obs & Pred & Obs & Pred & Obs & Pred & Obs & Pred \\
\hline$[\mathrm{S} / \mathrm{H}]$ & -2.26 & -2.34 & -0.63 & -0.76 & $\ldots$. & $\ldots$. & $\ldots$. & -1.27 \\
{$[\mathrm{Fe} / \mathrm{H}]$} & -2.54 & -2.50 & -1.64 & -1.81 & -1.78 & $\ldots$. & -2.26 & -2.22 \\
{$[\mathrm{C} / \mathrm{Fe}]$} & $>-0.91$ & -0.23 & $>-0.34$ & -0.42 & $\ldots$. & $\ldots$. & $\ldots$. & -0.32 \\
{$[\mathrm{~N} / \mathrm{S}]$} & -0.75 & -0.91 & $>-0.57$ & -0.79 & $\ldots$. & $\ldots$. & $\ldots$. & -0.88 \\
{$[\mathrm{~S} / \mathrm{Fe}]$} & +0.28 & 0.16 & +1.01 & 1.05 & $\ldots$. & $\ldots$. & $\ldots$. & 0.93 \\
{$[\mathrm{Si} / \mathrm{Fe}]$} & $>-0.28$ & 0.15 & $>+0.45$ & -0.01 & $>+0.72$ & $\ldots$. & $>+0.61$ & 0.09 \\
{$[\mathrm{O} / \mathrm{Fe}]$} & $>-0.91$ & 0.35 & $>-0.32$ & 0.07 & $\ldots$. & $\ldots$. & $\ldots$. & 0.34 \\
{$[\mathrm{Mg} / \mathrm{Fe}]$} & $<+0.90$ & 0.18 & +0.93 & -0.10 & $>-0.98$ & $\ldots$. & $>-0.97$ & 0.03 \\
{$[\mathrm{Ni} / \mathrm{Fe}]$} & -0.12 & -0.12 & +0.10 & -0.19 & -0.09 & $\ldots$. & -0.11 & -0.16 \\
{$[\mathrm{Zn} / \mathrm{Fe}]$} & $\ldots$. & -0.05 & +0.95 & 0.94 & $>+1.14$ & $\ldots$. & +0.65 & 0.74 \\
\hline \hline
\end{tabular}

Abundance ratios used in this work as measured in four GRB host galaxies and as predicted by means of our best models. All the abundances are normalized to the solar values, from Grevesse et al. (2007).

metallicity tracer (such as $[\mathrm{S} / \mathrm{H}]$ or $[\mathrm{Fe} / \mathrm{H}]$ ) can be used to constrain the nature and the star formation history of a given system, whereas the study of the abundance ratios 

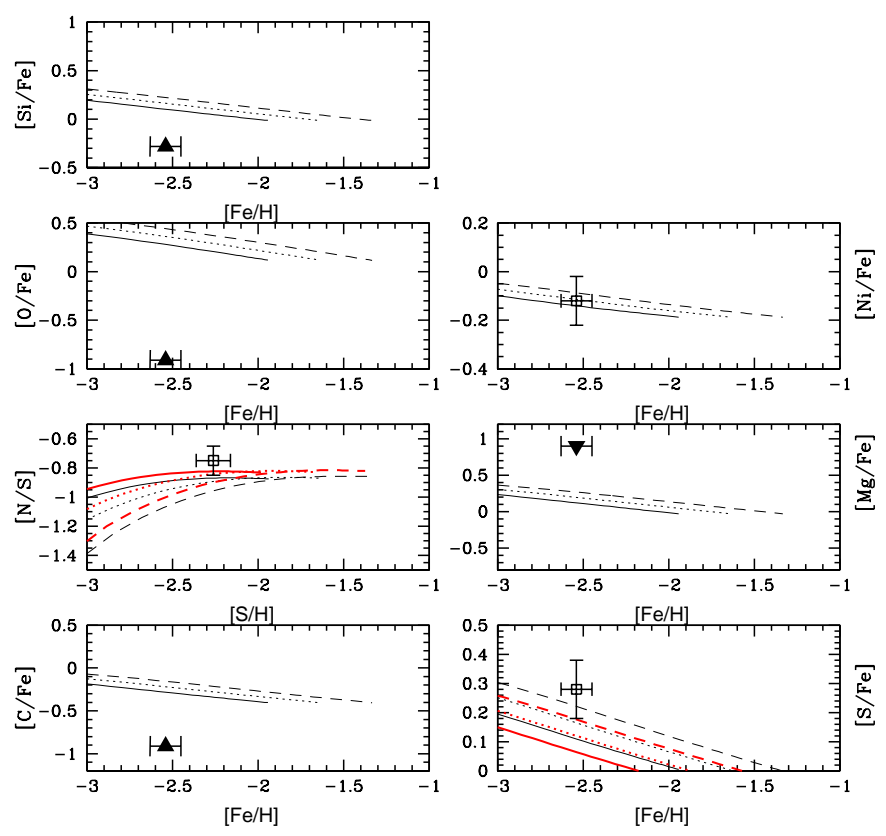

FIG. 2.- Observed abundance ratios versus metallicity for the host galaxy of GRB 050730 as derived by Prochaska et al. (2007a) (empty squares with error bars and solid triangles). The thin solid line, dotted line and dashed line are predictions computed by means of a chemical evolution model for a dwarf irregular galaxy assuming for the star formation efficiency $\nu=0.005 G y r^{-1}, \nu=0.01 G y r^{-1}$, and $\nu=0.02 G y r^{-1}$, respectively, with "expected dust" composition. The thick lines are as above, but with "alternative dust" composition, i.e. with S depleted.
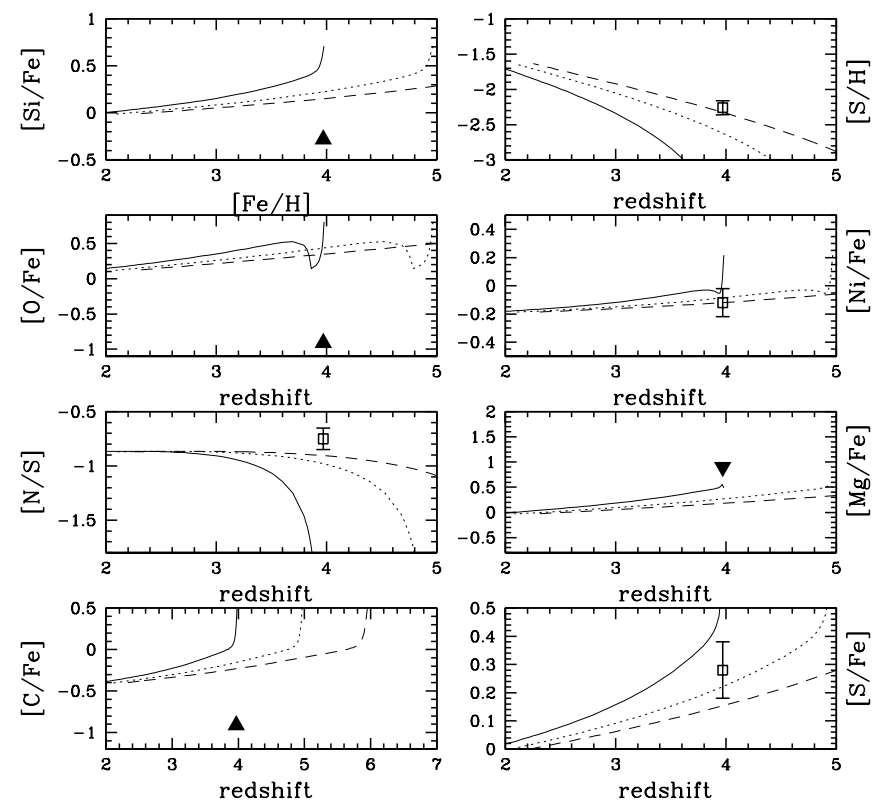

FIG. 3.- Observed abundance ratios versus redshift for the host galaxy of GRB 050730 as derived by Prochaska et al. (2007a) (empty squares with error bars and solid triangles). The thin solid line, dotted line and dashed line are predictions computed by means of the best model ( $\nu=0.01$ Gyr $^{-1}$, "expected dust" composition) and assuming $z_{f}=4, z_{f}=5$ and $z_{f}=6$, respectively. 
Calura et al.

versus redshift can be used to derive constraints on the age of the system.

In these 4 GRB DLAs, P07 have determined the abundance ratios for various elements, with typical errors of $\sim 0.1$ dex. For several other elements, only upper or lower limits were derived for the abundance ratios (see Tab. 1). We use the complete set of elements studied by P07 in order to constrain the SF history of the host galaxies of these 4 GRBs. In the following, we will present the results of our analysis, case by case.

\subsection{GRB 050730}

In this Section, our aim is to constrain the main parameter of the star formation history of the host galaxy of GRB 050730, i.e. its star formation efficiency $\nu$. We need to find the model which reproduces at best the observed abundance ratios.

In this study, the only parameter allowed to vary is the star formation efficiency. Its value is set by requiring that, with the adopted SF efficiency, the observed abundance ratios are reproduced.

We assume various values for the star formation efficiency $\nu$, spanning from $\nu_{\min }=0.005$ Gyr $^{-} 1$ to $\nu_{\max }=$ 0.1 Gyr $^{-} 1$. In figure 2 , we show the predicted evolution of various abundance ratios vs metallicity, traced either by $[\mathrm{S} / \mathrm{H}]$ or by $[\mathrm{Fe} / \mathrm{H}]$, calculated for a few models assuming different SF efficiency values and compared with the observed abundance ratios.

Any model with a star formation efficiency $\nu \leq$ $0.005 \mathrm{Gyr}^{-1}$ is rejected, since they match the abundances of the non-refractory element $N$ at ages larger than 1.53 Gyr, which is the age of the Universe corresponding to the redshift of the GRB $z_{G R B}=3.96$. In Fig. 2, the thin lines represent the predictions computed with the "expected dust" scenario, i.e. where $\mathrm{S}$ is undepleted whereas the thick lines are the predictions within the "alternative dust" scenario, where $\mathrm{S}$ is depleted.

Some of the shown observational abundance ratios are lower limits ([C/Fe, O/Fe] or upper limits $[\mathrm{Mg} / \mathrm{Fe}]$, and do not provide any strong constraint which can help us decide which model is the best in reproducing the data. Useful information come from the $[\mathrm{N} / \mathrm{S}]$ vs $[\mathrm{S} / \mathrm{H}]$ and $[\mathrm{S} / \mathrm{Fe}]$ vs $[\mathrm{Fe} / \mathrm{H}]$ plots. The $[\mathrm{N} / \mathrm{S}]$ vs $[\mathrm{S} / \mathrm{H}]$ plot tells us that, without taking into account $\mathrm{S}$ depletion, no model allows us to reproduce the observed [N/S] value. On the other hand, a small $\mathrm{S}$ depletion of $\sim 0.1$ dex is enough to have the observed $[\mathrm{N} / \mathrm{S}]$ reproduced by the two models with $\nu=0.005$ $\mathrm{Gyr}^{-1}$ and with $\nu=0.01 \mathrm{Gyr}^{-1}$. However, as explained in Sect. 2.2, our predicted [N/S] should be regarded as a lower limit, since the yields of Meynet \& Maeder (2002) for low and intermediate mass stars neglect the third dredge up, which may play an important role in $\mathrm{N}$ production (van den Hoek \& Groenewegen 1997).

The $[\mathrm{S} / \mathrm{Fe}]$ vs $[\mathrm{Fe} / \mathrm{H}]$ plot indicates that with $\mathrm{S}$ undepleted, the two models with $\nu=0.01 \mathrm{Gyr}^{-1}$ and $\nu=0.02 \mathrm{Gyr}^{-1}$ reproduce the observed $[\mathrm{S} / \mathrm{Fe}]$, however, once we take into account $\mathrm{S}$ depletion, only the model with $\nu=0.02 \mathrm{Gyr}^{-1}$ is consistent with the data. On the other hand, the $[\mathrm{Ni} / \mathrm{Fe}]$ vs $[\mathrm{Fe} / \mathrm{H}]$ plot does not allow us to disentangle among the different models, since models with various $\mathrm{SF}$ efficiencies present very similar $[\mathrm{Ni} / \mathrm{Fe}]$ values.

From the present study, we conclude that, within the "expected dust" scenario, i.e. neglecting any S depletion, the best model is the one with $\nu=0.01 \mathrm{Gyr}^{-1}$, with the small discrepancy between our predictions and the models possibly due to the fact that we are underestimating $[\mathrm{N} / \mathrm{H}]$ since we assume no $\mathrm{N}$ production in massive stars.

Within the "alternative dust" scenario, the best model is the one with $\nu=0.02 \mathrm{Gyr}^{-1}$. Any model with SF efficiency $\nu=0.03 \mathrm{Gyr}^{-1}$ or higher leads us to underestimate the $[\mathrm{N} / \mathrm{S}]$ value by $\sim 0.2$ dex. This can maybe reconciled by assuming primary $\mathrm{N}$ production in massive stars and more accurate yields for low and intermediate mass stars, including also the third dredge up. However, since a further investigation of the $\mathrm{N}$ is beyond the aim of the present paper, we think it is reasonable to reject any model underestimating $[\mathrm{N} / \mathrm{S}]$ by more than $0.2 \mathrm{dex}$, corresponding to the total error bar of the measured abundances considered here.

Our conclusion is that, within the "expected dust scenario", the best model is the one with with $\nu=0.01$ $\mathrm{Gyr}^{-1}$, whereas within the "alternative dust" scenario, the best model is the one with $\nu=0.02$.

Once we have constrained the SF efficiency of the GRB 050730 host galaxy, by studying its abundance ratios vs redshift we can derive constraints on its age. In the following, we will use the two best models of the "expected dust scenario" and "alternative dust scenario" to derive a value for the age of the host galaxy of the GRB.

In Fig. 3, we show the predicted redshift evolution of various abundance ratios, compared with the abundance ratios observed for GRB 050730. We show the predictions computed by means of the best model of the "expected dust " scenario by assuming three different values for the redshift of formation $z_{f}$, defined as the redshift at which the star formation turns on in the GRB host galaxy. The GRB host abundances are best reproduced by assuming $z_{f}=6$. The difference between $z_{f}$ and the redshift of the GRB host galaxy $z_{G R B}$ provides an estimate of the age of the system. The best model of the "expected dust" scenario points towards an age of $\sim 0.6$ Gyr. With the best model of the "alternative dust" scenario, a nearly identical value for the age of the GRB host can be derived.

At this age, the predicted specific star formation rate is 4.7 Gyr $^{-1}$, both for the best model of the "expected dust" and "alternative dust" scenario.

We have also a chance to test whether our assumption for dust evolution are appropriate, in particular the hypothesis of having neglected dust accretion. At the age of 0.6 Gyr, with the best model of the "expected dust" picture we predict a dust-to-gas ratio $D=1.2 \times 10^{-6}$, which is in very good agreement with the observational upper limit2 of Prochaska et al. (2007b) of $8 \times 10^{-6}$. The predicted dust-to-metals ratio is $D_{Z}=0.03$, again in agreement with the upper limit of 0.12 derived observationally by Prochaska et al. (2007b). Very similar values may be obtained by means of the best model of the "alternative dust" scenario. We conclude that the abundances of this system are not strongly influenced by dust depletion. Our assumptions concerning the treatment of dust evolution,

2 To compute the observed dust-to-gas and dust-to-metal ratios, we assume for the Small Magellanic cloud a $D_{S M C}=0.1 D_{\odot}=0.0008($ Issa et al. 1990, Rubio et al. 2004) and $Z_{\odot}=0.012$ (Grevesse et al. 2007). 

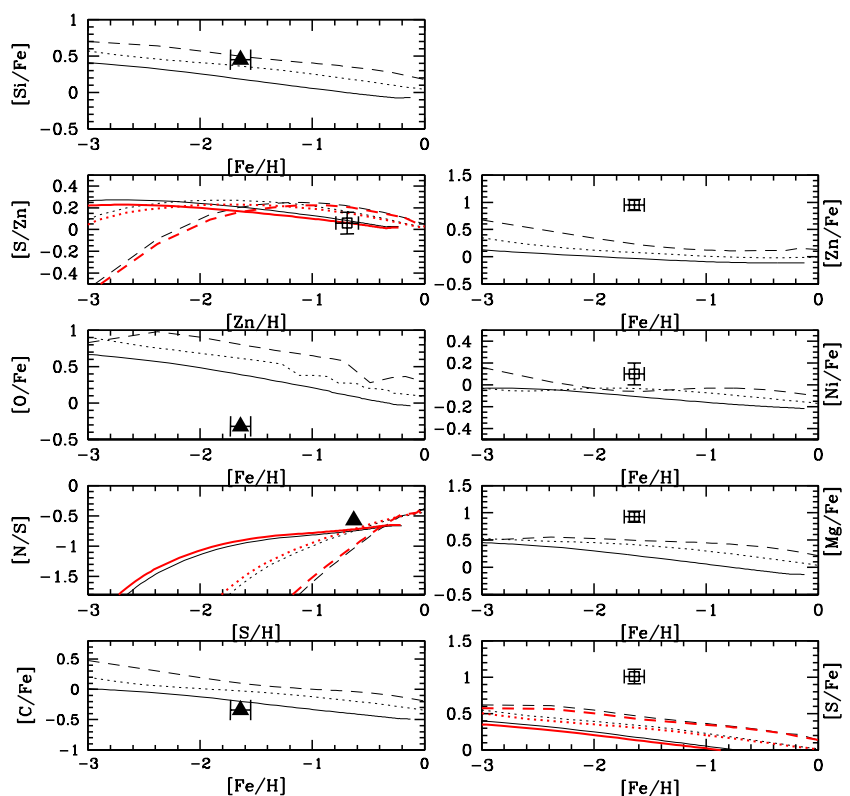

FIG. 4.- Observed abundance ratios versus metallicity for the host galaxy of GRB 050820 as derived by Prochaska et al. (2007a) (empty squares with error bars and solid triangles). The thin solid line, dotted line and dashed line are predictions computed by means of a chemical evolution model for a dwarf irregular galaxy assuming for the star formation efficiency $\nu=0.1$ Gyr $^{-1}, \nu=1 G^{-1}$, and $\nu=10 G y r^{-1}$, respectively, with "expected dust" composition. The thick lines are as above, but with "alternative dust" composition, i.e. with S depleted.
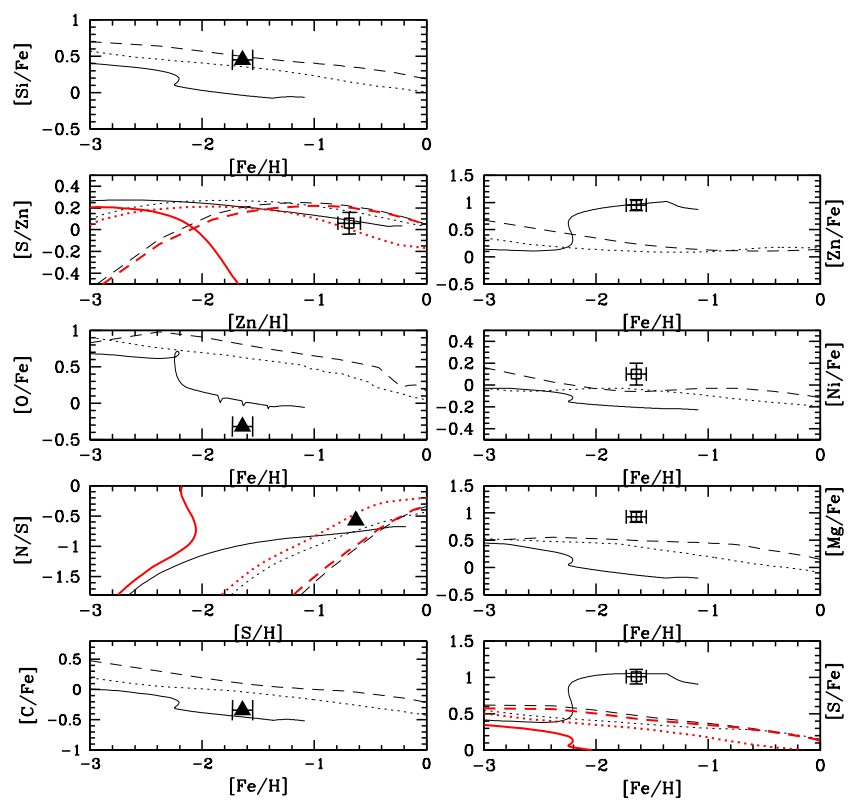

FiG. 5.- Symbols as in Fig 4. Thick and thin lines as in Fig 4, but including dust accretion. 

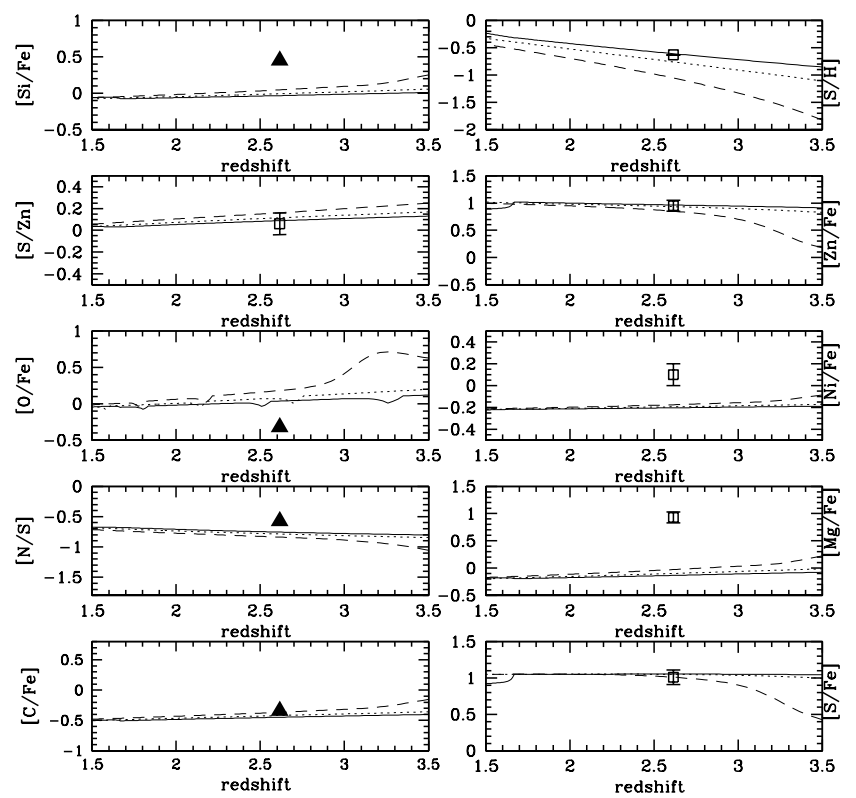

FIG. 6. - Observed abundance ratios versus redshift for the host galaxy of GRB 050820 as derived by Prochaska et al. (2007a) (empty squares with error bars and solid triangles). The dashed line, the dotted line and the solid line represent the predictions computed by means of the best model ( $\nu=0.1 \mathrm{Gyr}^{-1}$, dust accretion) within the "expected dust" scenario assuming $z_{f} \geq 4$, and $z_{f}=6$, and $z_{f}=10$, respectively.

in particular having neglected dust accretion in molecular clouds, seems appropriate for the study of this GRB host.

\subsection{GRB 050820}

In fig. 4, we show various observed interstellar abundance ratios vs metallicity for the host galaxy of GRB 050820. The observed abundance ratios are compared with predictions computed by means of three chemical evolution models with SF efficiencies $\nu=0.1 \mathrm{Gyr}^{-1}, \nu=1$ $\mathrm{Gyr}^{-1}$ and $\nu=10 \mathrm{Gyr}^{-1}$. In this case, we see how, with a model assuming only dust destruction and no dust accretion, the observed abundance ratios do not allow us to determine which model is the most accurate in reproducing the data. The predictions for the model with $\nu=0.1$ $\mathrm{Gyr}^{-1}$ allows us to reproduce the $[\mathrm{C} / \mathrm{Fe}],[\mathrm{O} / \mathrm{Fe}],[\mathrm{S} / \mathrm{Zn}]$ and are very close to the observed $[\mathrm{N} / \mathrm{S}]$ lower limit. However, no single model can reproduce the remaining set of abundance ratios shown in Fig. 4. In particular, the very high $[\mathrm{S} / \mathrm{Fe}],[\mathrm{Mg} / \mathrm{Fe}]$ and $[\mathrm{Zn} / \mathrm{Fe}]$ abundance ratios are not reproduced, even by assuming a star formation efficiency $\nu=10 \mathrm{Gyr}^{-1}$, a value generally used to describe elliptical galaxies (Calura, Matteucci \& Vladilo 2003). The same conclusion is valid taking into account $\mathrm{S}$ depletion (thick lines in Fig. 4). This GRB DLA presents peculiar abundance ratios, such as a high value for $[\mathrm{Zn} / \mathrm{Fe}]$ which, at such a $[\mathrm{Fe} / \mathrm{H}]$, in general is interpreted as the signature of dust depletion (see e.g. Vladilo 2004). To investigate the possibility that dust depletion may play a non negligible role in the ISM of the GRB 050820 host, we consider a chemical evolution model which takes into account dust accretion. To model dust accretion, the prescriptions are the same as described in CPM08. The dust accretion rate is $G_{d u s t, i} / \tau_{a c c r}$, where $\tau_{a c c r}$ is the dust accretion timescale

$$
\tau_{\text {accr }}=\tau_{0, i} /\left(1-f_{i}\right)
$$

where

$$
f_{i}=\frac{G_{d u s t, i}}{G_{i}}
$$

For the timescale $\tau_{0, i}$, typical values span from $\sim 5 \times 10^{7}$ $\mathrm{yr}$, of the order of the lifetime of a typical molecular cloud, up to $\sim 2 \times 10^{8}$ yr (Dwek 1998). In this paper, we assume that the timescale $\tau_{0, i}$ is constant for all elements, with a value of $5 \times 10^{7}$ yr (CPM08, Inoue 2003).

In Fig. 5, we show our results for the predicted abundance ratios of the three models with $\nu=0.1 \mathrm{Gyr}^{-1}$, $\nu=1 \mathrm{Gyr}^{-1}$, and $\nu=10 \mathrm{Gyr}^{-1}$ taking into account also dust accretion. By including accretion, a larger number of abundance ratios is now reproduced by the model with $\nu=0.1$, computed assuming no $\mathrm{S}$ depletion (thin lines in Fig. 5). In particular, the $[\mathrm{S} / \mathrm{Fe}]$ and $[\mathrm{Zn} / \mathrm{Fe}]$ ratios are now reproduced by the model with $\nu=0.1 \mathrm{Gyr}^{-1}$. The very high $[\mathrm{Si} / \mathrm{Fe}],[\mathrm{Mg} / \mathrm{Fe}]$ and $[\mathrm{Ni} / \mathrm{Fe}]$ are not reproduced by the same model, and they are likely to be due to differential depletion effects. Differential depletion is the most likely explanation for peculiar $[\mathrm{Si} / \mathrm{Fe}]$ and $[\mathrm{Mg} / \mathrm{Fe}]$ ratios observed also in some DLAs (Dessauges-Zavadsky et al. 2002, D07). In this paper, to limit the parameter space, we have assumed that all the chemical elements are incorporated into dust grains in the same proportions. A detailed modeling of differential depletion is beyond the aims of the present work. Future investigation on this topic, also in connection to dust depletion in DLAs, is required to shed light on the chemical species interested by differential depletion.

From Fig. 5, we see that, in presence of dust accretion, the predicted abundance ratios between a non-refractory element and a refractory one, such as $[\mathrm{Zn} / \mathrm{Fe}]$, are the highest for the model with the lowest SF efficiency. This counterintuitive result is due to the fact that, given the low con- 
densation efficiencies assumed here, with high star formation efficiencies $\left(\nu \geq 1 \mathrm{Gyr}^{-1}\right)$, dust grains hardly survive in the ISM even if accretion is present. This result is due to the fact that a high star formation efficiency implies a high dust destruction rate, which the process of accretion is not able to compensate. This is consistent with the results of CPM08, who found that dust destruction by SNe is more efficient than dust production. However, CPM08 assumed the same dust condensation efficiencies as Dwek (1998), considerably higher than the ones used in the present paper (e.g., for Si and Fe, CPM08 assumed $\delta_{S i, F e}=0.8$ ). In this case, also a galaxy with a SF efficiency of $\sim 10 \mathrm{Gyr}^{-1}$, such as an elliptical galaxy of luminous mass $10^{11} M_{\odot}$, can present a very high dust fraction, up to $0.9-1$, in the period when star formation is active.

It is also interesting to note that, in the "alternative dust" scenario, including $\mathrm{S}$ depletion and accretion, no model provides a satisfactory fit to the observed data.

Regarding GRB 050820, we conclude that the model with SF efficiency $\nu=0.1 \mathrm{Gyr}^{-1}$, "expected dust" composition and dust accretion is the best in reproducing the observed abundance ratios vs metallicity. We now use this model to infer the age of the GRB 050820 host galaxy.

In Fig. 6, we show various abundance ratios vs redshift as measured by P07 for GRB 050820, compared to our predictions computed by means of the best model and assuming three different redshifts of formation: $z_{f}=4, z_{f}=6$, and $z_{f}=10$. It is clear that the majority of the abundance ratios, i.e. all the abundance ratios except $[\mathrm{N} / \mathrm{S}]$, $[\mathrm{Si} / \mathrm{Fe}],[\mathrm{Mg} / \mathrm{Fe}]$ and $[\mathrm{Ni} / \mathrm{Fe}]$, all underestimated for the reasons described above, may be reproduced by assuming $z_{f} \geq 6$. In this way, we are able to derive a lower limit of 1.5 Gyr for the age of the host galaxy of GRB 050820. This allows us to derive an upper limit of 2.5 $\mathrm{Gyr}^{-1}$ for the SSFR. Now, we investigate whether our assumptions concerning dust provide a realistic description of the dust content of this GRB DLA. For the GRB 050820 DLA, Prochaska et al. (2007b) measure a dust-to-gas ratio 0.0008. Our results imply that, at the ages larger or equal to $1.5 \mathrm{Gyr}$, the predicted dust-to-gas ratio for the best model is $\geq 0.00057$, in very good agreement with the observed value. The predicted dust-to-metals ratio is 0.73 . By assuming for the observed metallicity $[\mathrm{Z} / \mathrm{H}]=[\mathrm{S} / \mathrm{H}]=-$ $0.67 \pm 0.1$ and by neglecting te uncertainty in the observed dust-to-gas, the measured dust-to-metals ratio is between 0.23 and 0.36 . Given the uncertainties of the parameters involved in our computation, in particular the dust condensation efficiencies and the accretion timescale, which may even be larger by a factor of 2 than the value we have adopted, i.e. $\tau_{0}=0.05 \mathrm{Gyr}$, we think our models provides a reasonable description of the dust properties of GRB 050820 .

\section{4. $G R B 051111$}

Of the elements studied in this paper, for GRB 051111, only 4 measured abundance ratios are available (see Tab. 1). However, of these 4, 3 are lower limits and only one is a real measure. In fig. 7, we show the observed interstellar abundance ratios vs metallicity for the host galaxy of GRB 051111, compared with predictions computed by means of three chemical evolution models with SF efficiencies $\nu=0.1 \mathrm{Gyr}^{-1}, \nu=1 \mathrm{Gyr}^{-1}$ and $\nu=10 \mathrm{Gyr}^{-1}$.
The predictions are shown both taking into account dust accretion (thick lines) and not (thin lines). The poor number of abundance ratios measured for this GRB DLA does not allow us to constrain its star formation history. However, the very large $[\mathrm{Zn} / \mathrm{Fe}]$ is indicative of strong dust depletion. Unfortunately, no measures are available for the dust-to-gas and dust-to-metals ratios, hence it is not possible to assess accurately the amount of dust depletion characterizing this system.

\subsection{GRB 060418}

Also For GRB 060418, only 4 measured abundance ratios are available to be compared with our predictions. In fig. 8, we show the observed interstellar abundance ratios vs metallicity for the host galaxy of GRB 060418, compared with predictions computed by means of three chemical evolution models with SF efficiencies $\nu=0.1$ $\mathrm{Gyr}^{-1}, \nu=1 \mathrm{Gyr}^{-1}$ and $\nu=10 \mathrm{Gyr}^{-1}$, taking into account dust accretion (thick lines) and not (thin lines). In this case, we have two real measures and two lower limits. Also this system presents a supersolar $[\mathrm{Zn} / \mathrm{Fe}]$ ratio, which is likely indicating the presence of dust depletion. The two actual measures allow us to derive some constraints on the star formation history and on the age of the host galaxy of GRB 060418. From Fig. 8, we see that the model which best reproduces the observed abundance ratios is the one characterized by $\nu=0.1 \mathrm{Gyr}^{-1}$ and including dust accretion. With this model, it is possible to reproduce two measured abundance ratios, the lower limit for $[\mathrm{Mg} / \mathrm{Fe}]$ is compatible with our predictions, whereas the $[\mathrm{Si} / \mathrm{Fe}]$ is not, probably owing to the effects of differential depletion, as explained above. We use this model to derive a value for the age and for the SSFR of the host galaxy of this GRB.

In Fig. 9, we show the measured abundance ratios vs redshift, compared to our predictions computed with the best model, assuming three different values for the redshift of formation $z_{f}$ : $z_{f}=1.6, z_{f}=1.8$ and $z_{f}=2$.. The observed abundance ratios are reproduced by assuming $z_{f}=1.8$. The redshift of the GRB is $z_{G R B}=1.5$, which, with the cosmology adopted in this paper, implies an age of $0.63 \mathrm{Gyr}$ and a SSFR of $2.8 \mathrm{Gyr}^{-1}$. At this age, we predict a dust-to-gas ratio of 0.0005 and a dust-to-metals ratio of 0.71 . Unfortunately, no observational value for the dust-to-gas ratio is available for this system.

A summary of the predicted physical properties of the GRB host galaxies studied in this paper is presented in Tab. 2.

\subsection{Specific star formation rates and implications for cosmic chemical evolution studies}

The specific star formation rate (SSFR) is defined as the ratio between the SFR and the stellar mass. Its determination does not require any a-priori assumption on unknown quantities involved in our study, such as the mass or the physical dimension of the system, which on the other hand have to be assumed in order to provide an estimate of the SFR or the SFR surface density.

The SSFR provides an indication of the intensity of the star formation of the host galaxy, whereas the inverse of the SSFR represents the star formation timescale, hence 


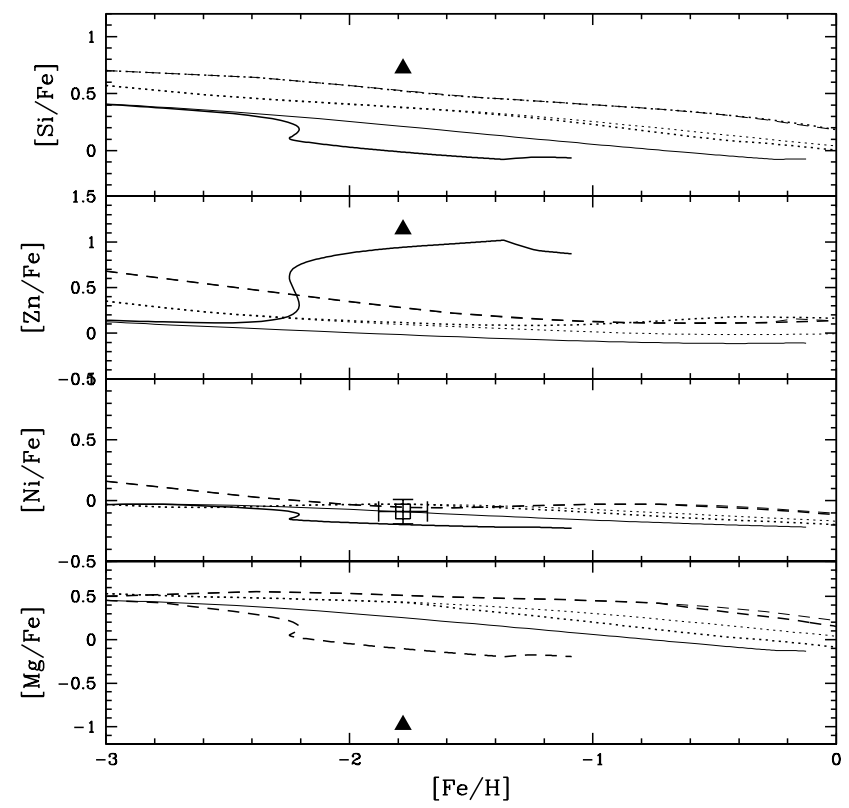

FIG. 7.- Observed abundance ratios versus metallicity for the host galaxy of GRB 051111 as derived by Prochaska et al. (2007a) (empty squares with error bars and solid triangles). The thin solid line, dotted line and dashed line are predictions computed by means of a chemical evolution model for a dwarf irregular galaxy assuming for the star formation efficiency $\nu=0.1 G_{y r^{-1}}, \nu=1 G y r^{-1}$, and $\nu=10 G y r^{-1}$, respectively, with "expected dust" composition. The thick lines are as above, but taking into account dust accretion

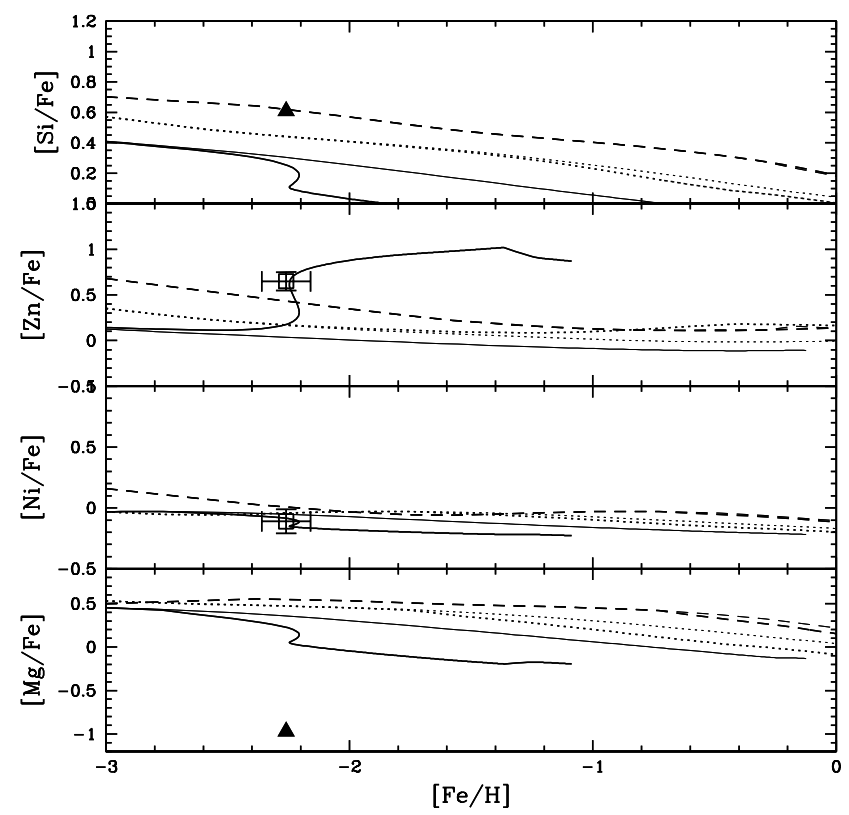

FIG. 8.- Observed abundance ratios versus metallicity for the host galaxy of GRB 060418 as derived by Prochaska et al. (2007a) (empty squares with error bars and solid triangles). The thin solid line, dotted line and dashed line are predictions computed by means of a chemical evolution model for a dwarf irregular galaxy assuming for the star formation efficiency $\nu=0.1 G_{y}^{-1}, \nu=1 G y r^{-1}$, and $\nu=10 G y r^{-1}$, respectively, with "expected dust" composition. The thick lines are as above, but taking into account dust accretion 


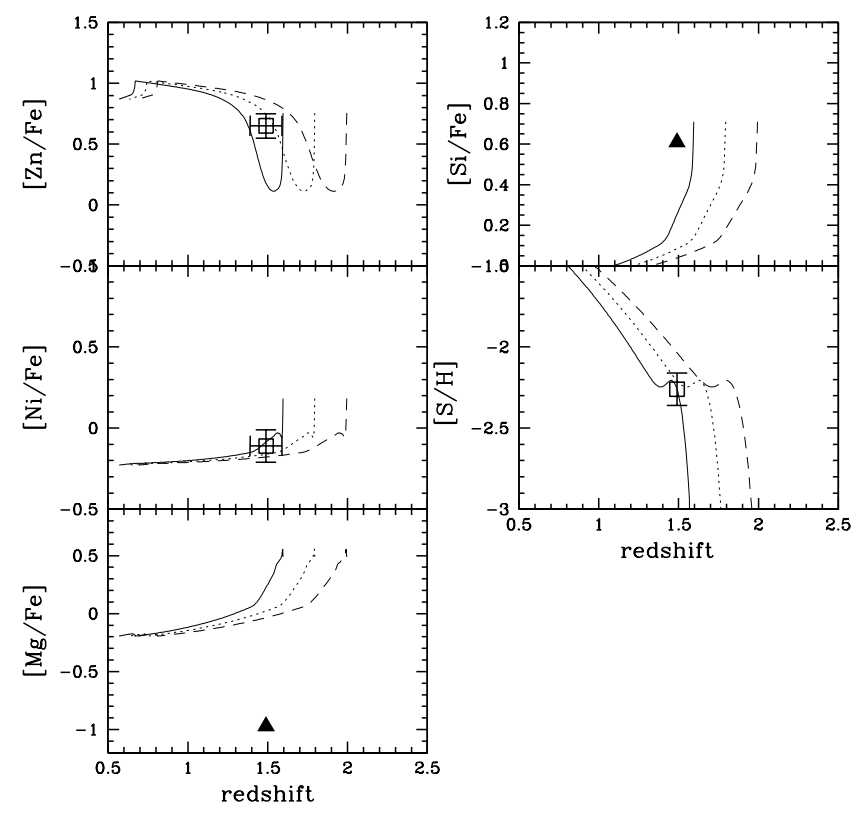

FIG. 9.- Observed abundance ratios versus redshift for the host galaxy of GRB 060418 as derived by Prochaska et al. (2007a) (empty squares with error bars and solid triangles). The solid line, the dotted line and the dashed line represent the predictions computed by means of the best model $\left(\nu=0.1 \mathrm{Gyr}^{-1}\right.$, dust accretion) within the "expected dust" scenario assuming $z_{f} \geq 1.6$, and $z_{f}=1.8$, and $z_{f}=2$, respectively.

a high value for the SSFR is associated to a young galaxy (Castro Cerón et al. 2006) . It is interesting to compare the SSFR values predicted by means of our models with the SSFR observed for a set of GRB host galaxies at redshifts $0<z<2.7$ by Castro Cerón et al. (2008). Castro Cerón et al. (2008) used the Spitzer images of 30 GRB hosts and estimated their stellar masses and their unobscured star formation rates by means of their K-band fluxes and rest-frame UV spectra, respectively. By means of these data, it has been possible to determine the SSFRs for the host galaxies of their sample. In the left panel of Fig. 10, we show the SSFR as a function of the stellar mass for two models with SF efficiency $\nu=0.01 \mathrm{Gyr}^{-1}$ (solid lines) and $\nu=0.1 \mathrm{Gyr}^{-1}$ (dashed lines). These models have turned out to be the best in describing the chemical abundance patterns of the GRB DLAs studied in this paper. The models shown in Fig. 10 assume various values for the mass normalization $M_{t o t}: M_{t o t}=10^{9} M_{\odot}$ (thin lines) and $M_{t o t}=10^{11} M_{\odot}$ (thick lines). This stellar mass range brackets the values observed in the bulk of the data by Castro Cerón et al. (2008). The models are compared to the data by Castro Cerón et al. (2008).

Until the onset of the galactic wind, each pair of models with the same SF efficiency plotted in Fig. 10 is characterized by the same chemical evolution and by the same abundance ratios. This is due to the fact that in chemical evolution models, to a first approximation, the abundance ratios do not depend on the total mass of the system, which plays a key role only in the presence of outflows.

The predictions shown in Fig. 10 have been computed until the present time, corresponding to $\sim 13.5$ Gyr for the cosmology adopted in the present paper. This is done to be consistent with the fact that the redshift range spanned by the data by Castro Cerón et al. (2008) is large, en- compassing $\sim 82 \%$ of the cosmic time. Several observational values plotted in Fig. 10 represent upper limits for the stellar mass and lower limits for the SSFR. In fact, Castro Cerón et al. (2008) showed that dust extinction must be present in at least $25 \%$ of the GRB hosts, causing the SFR determinations derived from UV/optical observations to be regarded as lower limits. The SSFR values by Castro Cerón et al. (2008) should be regarded as lower limits to the actual values also owing to a "dilution" effect present in mid-infrared photometry studies. This effect is related to the fact that some hosts of the sample are not spatially resolved, hence, the SSFRs are computed by dividing the UV SFRs by the total stellar masses, instead of dividing the SFRs by the stellar masses of the star forming regions, which are likely to encompass a small part of the host galaxy. For these reasons, if the corrections for dust extinction and dilution were performed and if the stellar masses were determined with higher precision, at least half of the data of Castro Cerón et al. (2008) should move towards our predictions.

In Fig. 10, right panel, we show the redshift evolution of the SSFR for our models, and for the GRB host galaxies as observed by Castro Cerón et al. (2008). In this case, in all our models, we have assumed that star formation begins at the same redshift $z=5$. Some of the predictions for the various models coincide across a large redshift interval. This is due to the fact that the SSFR is not dependent on the SF efficiency $\nu$ since, to a first approximation, both the SFR and the stellar mass are proportional to the SF efficiency $\nu$. In some cases, the predictions diverge owing to the onset of a galactic wind. This event concerns the models with the lowest mass normalizations, represented by the two thin lines in the right panel of Fig. 10, characterized by the shallowest gravitational potentials. As soon 
as the galactic wind develops, a considerable amount of gas becomes unavailable for star formation. The time the wind develops depends on the mass of the system and on the star formation efficiency $\nu$. In general, the higher is the adopted $\nu$ and the lower is the total mass, the earlier the wind develops. For this reason, after the onset of the wind, the SSFR does depend on the adopted star formation efficiency $\nu$.

According to our predictions, the SSFRs decrease with decreasing redshift. Our result in not surprising, since any galactic evolution model predicts an increase of the stellar mass and a decrease of the SFR with decreasing redshift. This is in agreement also with the observed cosmological evolution of the SFR, in particular with the observed decline of the cosmic star formation history with redshift at $z<2$ (Hopkins\& Beacom 2006), likely due to progressive gas consumption in late-type spiral discs (Calura \& Matteucci 2003, 2006). Furthermore, Papovich et al. (2006) showed that the integrated specific SFR, defined as the ratio between the comoving SFR density and the comoving stellar mass density, is a decreasing function of reshift. These results are apparently in contrast with the observed redshift evolution of the SSFR in GRB host galaxies. In fact, the data by Castro Cerón et al. (2008) suggest an increase of the SSFRs with decreasing redshift (see the solid straight line plotted in 10), at variance with any results concerning the redshift evolution of star forming galaxies at redshifts $0 \leq z \leq 3$. As stated also by Castro Cerón et al. (2008), the GRB host galaxies with the most vigorous star formation are likely to be heavily dust obscured, preventing the localization of their afterglows. This is one possible reason why, in the right panel of Fig. 10, no objects lay in the right upper corner of the plot. In the future, it will be important to extend the samples of GRB hosts with known star formation and stellar mass measures, in order to understand the reason for this apparent discrepancy and understand whether this trend is peculiar to GRB hosts or due to some selection effect. Surveys are ongoing to comprise GRB host galaxy samples based on localizations using the X-ray afterglow which is less susceptible to dust (Fynbo et al. 2007).

\subsection{A Comparison with SSFRs of Quasar DLAs}

In this section, we compare the SSFR of our models for GRB hosts with the values determined by means of the chemical evolution models for QSO DLAs presented by Dessauges-Zavadsky et al. (2007). We focus on the set of dwarf irregular galaxy models characterized by a continuous star formation history, which best reproduce the abundances of the set of DLAs studied by Dessauges-Zavadsky et al. (2007). By means of these models, the SSFRs are calculated at the time corresponding to the age predicted for the DLAs. In this way, for the 5 DLAs towards QSOs Q B0841+129, PKS $1157+014$, Q B1210+175, Q B2230+02, Q B2348-1444, we find SSFR between $0.24 \mathrm{Gyr}^{-1}$ and $7 \mathrm{Gyr}^{-1}$. In Table 3 , we show the SSFR values computed for the 5 DLAs of Dessauges-Zavadsky et al. (2007), compared to the values obtained for the hosts of GRB 050730. The SSFR computed for the DLAs studied by Dessauges-Zavadsky et al. (2007) are in substantial agreement with independent estimates for a larger sample of DLAs analysed by
Henry \& Prochaska (2007).

Although the technique used by Dessauges-Zavadsky et al. (2007) to correct the observations for dust depletion is different than the ones used here, it is interesting to note that the SF efficiencies of the models used to describe the abundances of QSO DLAs range from 0.05Gyr ${ }^{-1}$ to $0.1 \mathrm{Gyr}^{-1}$ (see Table 7 of Dessauges-Zavadsky et al. 2007), i.e. basically similar to the values used here. A quantitative analysis of the differences in the dust depletion corrections computed here and the ones used by Dessauges-Zavadsky et al. (2007) is beyond the scope of the present paper, but it is certainly an interesting subject for future studies.

It is may be interesting to compare the SSFR values for the QSO DLAs of D07 with the values found in this paper for GRB DLAs. The abundances of the DLAs studied by D07 required various depletion corrections. To correct for dust depletions, D07 used the technique described by Vladilo (2004). The implied depletion corrections (considering the $[\mathrm{Fe} / \mathrm{H}]$ abundance) were 0.14 dex for QB0841+129 at $\mathrm{z}=2.375,0.28$ dex for PKS1157+014, 0.14 dex for QB1210+175, 0.33 dex for QB2230+02 and 0.12 dex for QB2348-1444.

The approach to deal with dust depletion used here is different than the one by D07. However, the two approaches are consistent, since both predict high $[\mathrm{Zn} / \mathrm{Fe}]$ ratios for extremely dusty systems, and solar or nearly solar $[\mathrm{Zn} / \mathrm{Fe}]$ for dust-free systems. Moreover, possible depletion effects involving $\mathrm{S}$ do not influence our results (see Sect. 3.2).

It is probably wise and cautious to compare the SSFRs obtained for the three most dust poor DLAs of D07, with the star formation history derived here for GRB 050730, for which both the observations and our predictions indicate a very low dust content. To perform the comparison, we choose the SSFRs derived for QB0841+129 at $\mathrm{z}=2.375$, QB1210+175 and QB2348-1444. The values found for these 3 QSO DLAs of Dessauges-Zavadsky et al. (2007) are plotted in Fig. [11. As can be seen from Fig. 11] the SSFR of these 3 QSO DLAs are higher than the SSFRs of the best models of the "expected dust" scenario (within the "alternative dust" scenario, a very similar curve is obtained). The majority of QSO-DLA sightlines do not penetrate highly SF galaxies, being the most metal rich, most vigorously star-forming systems very rare in QSO DLA samples, possibly owing to dust obscuration of the background quasars (Hopkins \& Beacom 2006; Vladilo et al. 2008). Current expectation is that QSODLAs, which are drawn according to HI cross-section, most frequently correspond to sub- $L_{*}$ galaxies, as evidenced by their significantly sub-solar metallicities (e.g. Fynbo et al. 2008). At high $\mathrm{z}$, the luminosity function is sufficiently steep that sub- $L_{*}$ galaxies dominate the integrated SFR (Fynbo et al. 2008). For this reason, DLA samples should be able to trace the bulk of star formation. Prochaska et al. (2007a) discuss how GRB DLAs preferentially probe gas associated to the innermost regions of galaxies. This gas is generally denser and more metal rich than the gas probed by QSO DLAs which, on the other hand, are likley to arise in the outer galactic regions. If GRB DLAs probe regions of the Universe different than the ones of QSO DLAs, the GRB DLA and QSO DLA 
TABLE 2

Summary of the Physical properties of the 4 GRB host GalaXies STUdied in the PRESENT PAPER

\begin{tabular}{ccccc}
\hline \hline GRB & Age $($ Gyr $)$ & SSFR $\left(\right.$ Gyr $\left.^{-1}\right)$ & Dust-to-gas & Dust-to-metals \\
050730 & 0.61 & 4.7 & $1.2 \times 10^{-6}$ & 0.03 \\
050820 & $\geq 1.53$ & $\leq 2.5 \mathrm{Gyr}^{-1}$ & $\geq 0.00057$ & 0.73 \\
051111 & - & - & - & - \\
060418 & 0.66 & - & 0.0005 & 0.71 \\
\hline \hline
\end{tabular}

TABLE 3

Specific Star Formation Rates predicted for GRB hosts and QSO DLAs

\begin{tabular}{lc}
\hline \hline \multicolumn{1}{c}{ DLA } & SSFR $\left(\right.$ Gyr $\left.^{-1}\right)$ \\
\hline QB0841+129 & 1.5 \\
PKS1157+014 & 0.57 \\
QB1210+175 & 0.48 \\
QB2230+02 & 0.24 \\
QB2348-1444 & 7. \\
\hline \multicolumn{1}{c}{ GRB } & SSFR $\left(\right.$ Gyr $\left.^{-1}\right)$ \\
\hline GRB 050730 & 4.7 \\
GRB 050820 & $\leq 2.5$ \\
GRB 050820 & $\leq 2.8$ \\
\hline \hline
\end{tabular}
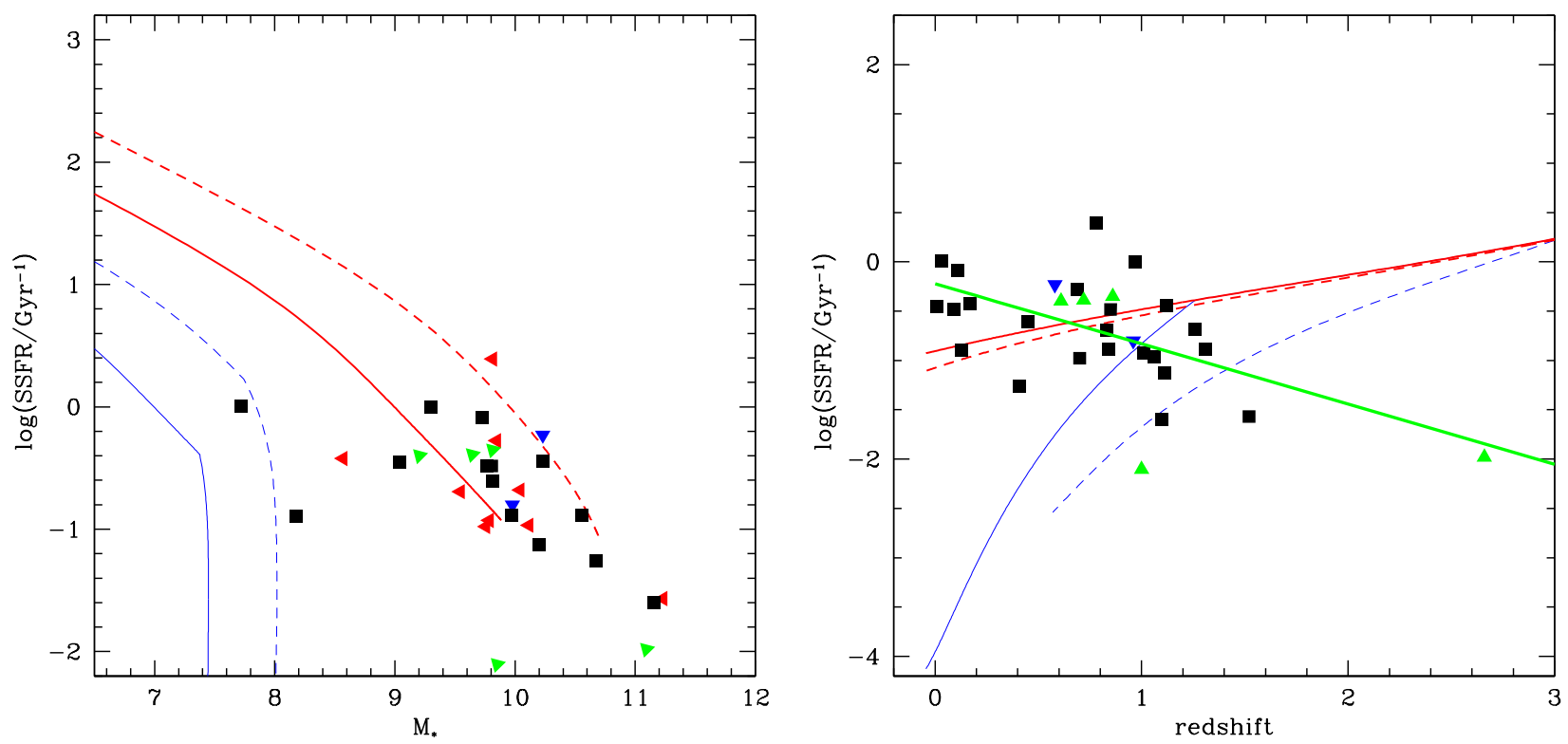

FIG. 10. - (a): SSFR as a function of the stellar mass for two models with SF efficiency $\nu=0.01 \mathrm{Gyr}^{-1}$ (solid lines) and $\nu=0.1 \mathrm{Gyr}-1$ (dashed lines), and assuming $M_{t o t}=10^{9} M_{\odot}$ (thin lines) and $M_{t o t}=10^{11} M_{\odot}$ (thick lines). The solid squares and the triangles are the observational determinations by Castro Cerón et al. (2008). The triangles mark lower or upper limits for the derived stellar mass, SSFR or both. (b): Redshift evolution of the SSFR for our models, and for the GRB host galaxies as observed by Castro Cerón et al. (2008). All the curves, squares and triangles are as in panel $a$. The thick dot-dashed line represents the best fit to the observed values, computed by means of a straight line $y=a x+b$. All the other curves, squares and triangles are as in panel $a$.

samples may provide complementary information on cosmic chemical evolution.

\section{CONCLUSIONS}

In this paper, for the first time we have used the elemental abundances observed in the host galaxy of 4 long
GRBs to constrain their star formation history and its age. Our method is based on the simultaneous study of the abundance ratios between various elements synthesized by stars on different timescales, by comparing the measured abundance ratios to the predictions of a detailed chemical evolution model. From the simultaneous study of the 


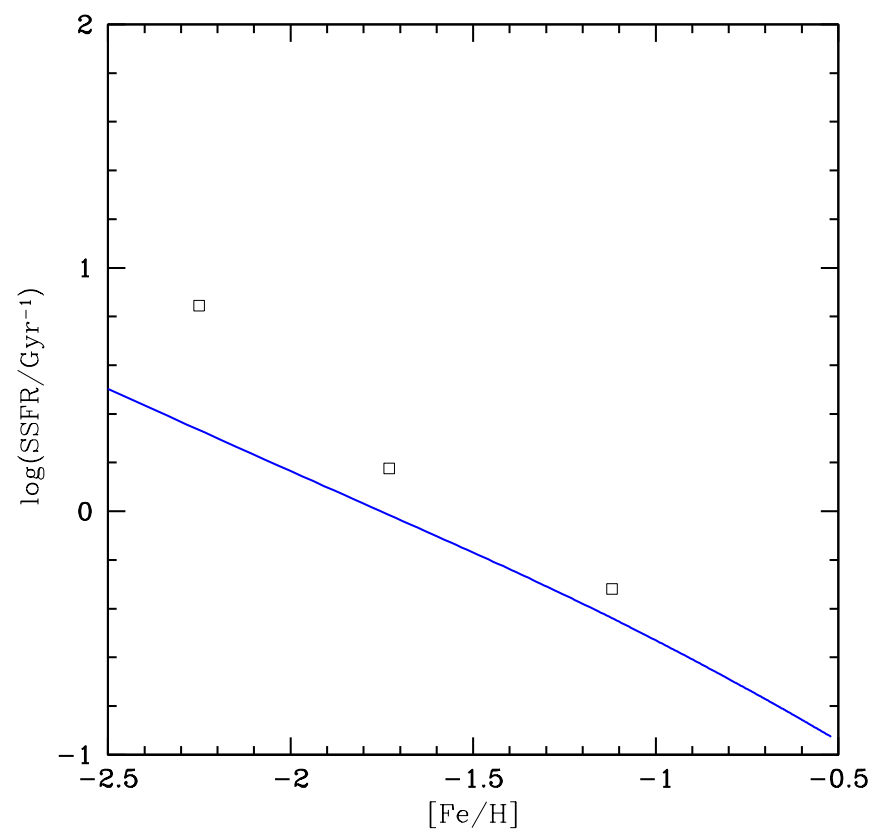

FIG. 11. - Predicted evolution of the SSFR vs $[\mathrm{Fe} / \mathrm{H}]$ for the best model for GRB 050730 host galaxy. The open squares are the values determined for the three QSO DLAs of the sample by Dessauges-Zavadsky et al. (2007) presenting the lowest dust depletions.

abundance ratios between various elements vs metallicity (traced by $[\mathrm{Fe} / \mathrm{H}],[\mathrm{S} / \mathrm{H}]$ or $[\mathrm{Zn} / \mathrm{H}]$ ), we have been able to constrain the star formation history of some of the host galaxies. By studying the abundance ratios vs redshift, we have been able to constrain the redshift of formation and the age of the GRB host galaxies. We tested models both with and without dust accretion. Some peculiar abundance ratios, such as very high $[\mathrm{Zn} / \mathrm{Fe}]$ ratios, seem to indicate severe dust depletion and, to be explained, require the inclusion of dust accretion in the models. Our main results can be summarized as follows:

1) Our results point towards star formation efficiencies between $0.01 G y r^{-1}$ and $0.1 G y r^{-1}$, ages for the system between 0.6 and $\geq 1.5$ Gyr. The observed dust content of some GRB DLAss is also satisfactorily accounted for.

2) The predicted specific star formation rate values are between 2.5 Gyr ${ }^{-1}$ and $4.7 G_{y r}{ }^{-1}$. These values are compatible with observational estimates from a very recent compilation by Castro Cerón et al. (2008). Our models predict a decrease of the SSFR with redshift, consistent with the observed decrease of the comoving cosmic SFR density between $z \sim 2$ and $z=0$, in agreement with numerous observations of star forming galaxies at low and high redshift. On the other hand, apparently the detected GRB hosts follow an opposite trend in the SSFR vs redshift plot, with a slight increase of the SSFR with decreasing redshift. In the future, it will be important to asses whether this apparent trend is real or if it is due to some selection effect affecting the observation of GRB host galaxies.

3) The study of the abundance pattern of the host galaxy of GRB 050730 indicates that the predicted specific star formation rate is compatible with the values found for the the set of DLAs studied by Dessauges-Zavadsky et al.
(2007). This implies that, the host galaxy of GRB 050730 may have followed a chemical evolution path similar to the ones typical of QSO DLAs.

Our study favors the hypothesis that long duration GRBs occur preferentially in low metallicity, star forming galaxies, characterized by low star formation efficiencies $(\nu \leq$ $\left.0.1 \mathrm{Gyr}^{-1}\right)$.

The fact that GRBs occur preferentially in low metallicity systems is indicated also by the luminosity-metallicity relation (Wolf \& Podsiadlowski 2007) and by some progenitor models for GRBs, where low metallicity hampers large loss of angular momentum and mass and the progenitor can retain a rapid rotating core, as required in particular in the collapsar model for long GRBs (MacFadyen \& Woosley 1999).

In the future, in order to have a better understanding of the chemical evolution patterns observed in high-redshift galaxies, it will be important to refine the study of dust depletion in local and distant galaxies, in order to clarify whether elements such as $\mathrm{S}$ have a refractory nature or not.

Furthermore, it will be important to extend the study carried on in this paper to a larger set of GRB DLAs, in order to have an insight into regions of the universe complementary to those probed by QSO DLAs and by other types of astrophysical objects, detected by means of different techniques.

FC acknowledges financial contribution from contract ASI-INAF I/016/07/0.

J. X. P. is partially supported by NASA/Swift grants NNG06GJ07G and NNX07AE94G and an NSF CAREER grant (AST-0548180). 


\section{REFERENCES}

Berger, E.; Fox, D. B.; Kulkarni, S. R.; Frail, D. A.; Djorgovski, S. G., 2007, ApJ, 660, 504

Bloom, J. S.; Djorgovski, S. G.; Kulkarni, S. R.; Frail, D. A., 1998, ApJ, 507, L25

Bradamante, F., Matteucci, F., D'Ercole, A. 1998, A\&A,337, 338

Calura, F., Matteucci, F., 2003, ApJ, 596, 734

Calura F., Matteucci F., 2004, MNRAS, 350, 351

Calura F., Matteucci F., 2006, ApJ, 652, 889

Calura, F., Matteucci, F., \& Vladilo, G. 2003, MNRAS, 340, 59

Calura, F.; Pipino, A.; Matteucci, F., 2008, A\&A, 479, 669

Castro Cerón, J. M.; Michaowski, M. J.; Hjorth, J.; Watson, D.; Fynbo, J. P. U.; Gorosabel, J., 2006, ApJ, 653, L85

Castro Cerón, J. M.; Michaowski, M. J.; Hjorth, J.; Watson, D.; Fynbo, J. P., 2008 ApJ, in press, arXiv:0803.2235

Chen, H.-W., et al. 2005, ApJ, 634, L25

Chiappini, C., Matteucci, F., Romano, D., 2001, ApJ, 554, 1044

Chiappini, C., Matteucci, F., Meynet, G., 2003, A\&A, 410257

Chiappini, C., Matteucci, F., Ballero, S. K., 2005, A\&A, 437, 429

Chiappini, C.; Hirschi, R.; Meynet, G.; Ekstrm, S.; Maeder, A.; Matteucci, F., 2006, A\&A, 449, L27

Christensen, L.; Hjorth, J.; Gorosabel, J., 2004, A\&A, 425, 913

Clayton, Geoffrey C., Green, J., Wolff, Michael J., Zellner, Nicolle E. B., Code, A. D., Davidsen, Arthur F., WUPPE Science Team, HUT Science Team, 1996, ApJ, 460313

D'Elia, V., et al., 2007, A\&A, 467, 629

Dessauges-Zavadsky, M., Prochaska, J. X.; D'Odorico, S.; 2002, A\&A, 391, 801

Dessauges-Zavadsky, M.; Calura, F.; Prochaska, J. X.; D'Odorico, S.; Matteucci, F., 2004, A\&A, 416, 79

Dessauges-Zavadsky, M.; Prochaska, J. X.; D'Odorico, S.; Calura, F.; Matteucci, F., 2006, A\&A, 445, 93

Dessauges-Zavadsky, M.; Calura, F.; Prochaska, J. X.; D'Odorico, S.; Matteucci, F., 2007, A\&A, 470, 431 (D07)

Djorgovski, S. G.; Bloom, J. S.; Kulkarni, S. R., 2003, ApJ, 591, L13

Dwek E., 1998, ApJ, 501, 643 (D98)

Dwek, E.; Galliano, F.; Jones, A. P., 2007, ApJ, 662, 927

Edmunds, M. G., 2001, MNRAS, 328, 223

Fruchter, Andrew S.et al., 1999, ApJ, 519, L13

Fynbo, J. P. U, et al., 2006, A\&A, 451, L47

Fynbo, J. P. U, Hjorth, J.; Malesani, D.; Sollerman, J.; Watson, D.; Jakobsson, P; Gorosabel, J.; Jaunsen, A. O., 2007, to appear in the proceedings of the Eleventh Marcel Grossmann Meeting on General Relativity, eds. H. Kleinert, R. T. Jantzen \& R. Ruffini, World Scientific, Singapore, 2007, (arXiv:astro-ph/0703458)

Fynbo, J. P. U.; Prochaska, J. X.; Sommer-Larsen, J.; DessaugesZavadsky, M.; Moller, P., 2008, ApJ, accepted, arXiv0801.3273

Grevesse, N.; Asplund, M.; Sauval, A. J., 2007, SSRv, 130, 105

Henry, R. B. C.; Prochaska, Jason X., 2007, PASP, 119, 962

Hopkins, A. M., Beacom, J. F., 2006, ApJ, 651,142

Jones, A. P., Tielens, A. G. G. M., Hollenbach, D. J., McKee, C. F., 1994, ApJ, 433, 797

Keller, L. P.; Hony, S.; Bradley, J. P.; Molster, F. J.; Waters, L. B. F. M.; Bouwman, J.; de Koter, A.; Brownlee, D. E.; Flynn, G. J.; Henning, T.; Mutschke, H., 2002, Nature, 417, 148

Kimura H., Mann I., Jessberger E. K., 2003, ApJ, 582, 846

Issa M. R., MacLaren I., Wolfendale A. W., 1990, A\&A, 236, 237

Iwamoto, K.; Brachwitz, F.; Nomoto, K.; Kishimoto, N.; Umeda, H.; Hix, W. R.; Thielemann, F.-K., 1999, ApJS, 125, 439

Inoue A. K., 2003, PASJ, 55, 901

Lanfranchi, G., Matteucci, F., 2003, MNRAS, 345, 71

Le Floc'h, E.; Duc, P.-A.; Mirabel, I. F.; Sanders, D. B.; Bosch, G.; Rodrigues, I.; Courvoisier, T. J.-L.; Mereghetti, S.; Melnick, J., 2002, ApJ, 581, L81

Le Floc'h, E.; Charmandaris, V.; Forrest, W. J.; Mirabel, I. F.; Armus, L.; Devost, D., 2006, ApJ, 642, 636

Levesque, E. M.; Kewley, L. J., 2007, ApJ, 667, L121

Lisenfeld U., Ferrara A., 1998, ApJ, 496, 145
Lodders K., 2003, ApJ, 591, 1220

MacFadyen A. and Woosley S., 1999, ApJ, 524, 262

Matteucci, F., 1992, ApJ, 397, 32

Matteucci, F., 2001, The chemical evolution of the Galaxy, Astrophysics and space science library, Volume 253, Dordrecht: Kluwer Academic Publishers

Meynet, G., Maeder, A. 2000, A\&A, 361, 101

Meynet, G., Maeder, A. 2002, A\&A, 390, 561

Morgan H. L., Edmunds M. G., 2003, MNRAS, 343, 427

Matteucci F., 1986, MNRAS, 221, 911

McKee C. F., 1989, in Allamandola L. J., Tielens A. G. G. M., eds, Interstellar Dust, Proc. IAU Symposium 135. Kluwer, Dordrecht, p. 431

Meynet, G., Maeder, A., 2002, A\&A, 381, L25

Papovich, C., et al., 2006, in "Extreme Starbursts: Near and Far", editors: Yu Gao \& D. B. Sanders, in press, astro-ph/0601408

Penprase, B. E.; Berger, E.; Fox, D. B.; Kulkarnı, S. R.; Kadısh, S.; Kerber, L.; Ofek, E.; Kasliwal, M.; Hill, G.; Schaefer, B.; Reed, M., 2006, ApJ, 646, 358

Pettini, M.; Zych, B. J.; Steidel, C. C.; Chaffee, F. H., 2008, MNRAS, 385,2011

Phillips, J. P., 2007, MNRAS, 381, 117

Price, P. A., et al., 2007, ApJ, 663, L57

Prochaska, J. X.; Bloom, J. S.; Chen, H.-W.; Hurley, K. C.; Melbourne, J.; Dressler, A.; Graham, J. R.; Osip, D. J.; Vacca, W. D., 2004, ApJ, 611, 200

Prochaska, J. X.; Chen, H.-W.; Bloom, J. S.; Dessauges-Zavadsky, M.; O'Meara, J. M.; Foley, R. J.; Bernstein, R.; Burles, S.; Dupree, A. K.; Falco, E.; Thompson, I. B., 2007, ApJS, 168, 231

Prochaska, J. X.; Chen, H.-W.; Dessauges-Zavadsky, M.; Bloom, J. S., 2007, ApJ, 666, 267 (P07)

Ruffle, D. P.; Hartquist, T. W.; Caselli, P.; Williams, D. A., 1999, MNRAS, 306, 691

Rubio, M.; Boulanger, F.; Rantakyro, F.; Contursi, A., 2004, A\&A, 425, L1

Savage B. D., Sembach K. R., 1996, ARA\&A, 34, 279

Savaglio, S., Fall, S. M., Fiore, F., 2003, ApJ, 585, 638

Savaglio, S., Glazebrook, K., Le Borgne, D., 2008, ApJ, submitted , arXiv0803.271

Scappini, F.; Cecchi-Pestellini, C.; Smith, H.; Klemperer, W.; Dalgarno, A., 2003, MNRAS, 341, 657

Schmidt M., 1959, ApJ, 129, 243

Sembach, K. R.; Howk, J. C.; Ryans, R. S. I.; Keenan, F. P., 2000, ApJ, 528, 310

Spite, M., et al., 2005, A\&A, 430, 655

Starling, R. L. C., et al., 2005, A\&A, 442, L21

Sugerman, B. E. K., et al., 2006, Science, 313, 196

Tumlinson, J., Prochaska, J. X., Chen, H.-W., Dessauges-Zavadsky, M., Bloom, J. S. 2007, ApJ, 668, 667

van den Hoek L. B. \& Groenwegen M. A. T., 1997, A\&AS, 123, 305

Vladilo, G., 2004, A\&A, 421, 479

Vladilo, G.; Prochaska, J. X.; Wolfe, A. M., 2008, A\&A, 478, 701

Vreeswijk, P. M., et al., 2004, A\&A, 419, 927

W elty, D. E., Lauroesch, J. T., Blades, J. C., Hobbs, L. M., York, D. G. 1997, ApJ, 489, 672

Welty, D. E., Hobbs, L. M., Lauroesch, J. T., Morton, D. C., Spitzer, L., York, D. G. 1999a, ApJS, 124, 465

Welty, D. E., Frisch, P. C., Sonneborn, G., York, D. G. 1999b, ApJ, 512,636

Welty, D. E.; Lauroesch, J. T.; Blades, J. C.; Hobbs, L. M.; York, D. G., 2001, ApJ, 554, L75

Whalen, D., Prochaska, J. X., Heger, A., Tumlinson, J., 2008, ApJ, submitted

Wiersema, K., et al., 2007, A\&A, 464, 529

Wolf, C., Podsiadlowski, P., 2007, MNRAS, 375, 1049

Woosley, S.E., Weaver, T.A., 1995, ApJS, 101, 181

Zhukovska S., Gail H.-P., Trieloff M., 2007, A\&A, 479, 453 